\title{
Optimal Location, Sizing, and Appropriate Technology Selection of Distributed Generators for Minimizing Power Loss Using Genetic Algorithm
}

\author{
T. R. Ayodele, A. S. O. Ogunjuyigbe, and O. O. Akinola \\ Power Energy Machine and Drives (PEMD) Research Group, Electrical and Electronic Engineering Department, \\ Faculty of Technology, University of Ibadan, Ibadan 200284, Nigeria
}

Correspondence should be addressed to A. S. O. Ogunjuyigbe; aogunjuyigbe@yahoo.com

Received 10 June 2015; Accepted 2 September 2015

Academic Editor: Yongsheng Chen

Copyright (C) 2015 T. R. Ayodele et al. This is an open access article distributed under the Creative Commons Attribution License, which permits unrestricted use, distribution, and reproduction in any medium, provided the original work is properly cited.

\begin{abstract}
Genetic algorithm (GA) is utilized to select most suitable Distributed Generator (DG) technology for optimal operation of power system as well as determine the optimal location and size of the DG to minimize power loss on the network. Three classes of DG technologies, synchronous generators, asynchronous generators, and induction generators, are considered and included as part of the variables for the optimization problem. IEEE 14-bus network is used to test the applicability of the algorithm. The result reveals that the developed algorithm is able to successfully select the most suitable DG technology and optimally size and place the DGs to minimize power loss in the network. Furthermore, optimum multiple placement of DG is considered to see the possible impact on power loss in the network. The result reveals that multiple placements can further reduce the power loss in the network.
\end{abstract}

\section{Introduction}

Recent researches have revealed that installation of Distributed Generators (DGs) in the power network has some advantages [1] which include the improvement in voltage profile [2] and reduction in the power loss on the power network [3-5]. The extent to which DGs reduce power system loss and improves voltage profile depends on the size and location of the DGs $[6,7]$. The different modes through which DGs affect the reactive power in a network enable it to provide voltage support [8]. This support however depends on the deliberate placement and sizing of DG to improve the voltage profile of the network [9]. Hence, to maximize these benefits, it is crucial to find the optimal size of DGs and their appropriate locations in the network, as sitting of DG units in improper locations could jeopardizes the system operation [10].

Several models and methods have been suggested for the solution of the optimal sizing and location of DGs: selection of optimal location and sizing of multiple DGs have been performed by Kumar using Kalman Filter Algorithm [11]. It was similarly reported that the algorithm is effective for determining the size and location of DG. Moreover, it has the advantage in that it runs on fewer samples compared to other algorithms, thereby reducing the computational burden usually experienced during the optimization process. Rani and Davi [12] have optimally determined the location and the size of DG on IEEE 33-bus system using the exact loss formula approach. The result reveals that the method was able to achieve reduction in power losses and improved the voltage profile of the system. A novel algorithm which uses economic dispatch approach was developed by Kamel and Kermanshahi [13]. The algorithm was used to determine the optimum size and location of the DGs embedded in the distribution network. The algorithm also takes into account the power cost and the available rating of DGs if the DGs exist in a competitive market. The technique was applied to three test distribution systems with different sizes ( 6 buses, 18 buses, and 30 buses). The results indicated that if the DGs are located at their optimal locations and have optimal sizes the total losses in the distribution network will be reduced by nearly $85 \%$.

The optimum size and location of capacitors and distributed generations (DGs) are determined simultaneously in 
a radial distribution network in [14]. The objective function includes power losses reduction and voltage profile improvement using ant colony algorithm. The proposed method was tested on IEEE 33-bus test system. The results show a considerable reduction in the total power loss in the system and improved voltage profiles of all the buses. Similarly, Allocating of DGs and optimal locations and size of Solid State Fault Current Limiters (SSFCLs) have been implemented by Shahriari and Samet [15] using genetic algorithm (GA). Optimal placement and sizing of multiple distributed generation in radial distribution feeders have been performed by Nagireddy et al. [16] using combined differential evaluation, HPSO method, with the objective of reducing the real power loss and improving the voltage profile of the network. Backtracking Search Algorithm has been used by Ishak et al. [17] for optimal DG placement and sizing for voltage stability improvement and power loss reduction. The applicability of the proposed method was verified using the IEEE 30bus transmission network. It was revealed that the proposed method is effective in optimally sizing and locating DG in distribution systems.

Genetic algorithm (GA) has been proposed by Kotb et al. [18] for optimum sizing and placement of DGs in a distributed network. The total active and reactive power losses were minimized and voltage profile was improved. GA fitness function introduced includes the active power losses, reactive power losses, and the cumulative voltage deviation variables. It was argued that GA can be used as a better tool than traditional methods to enable the planners to choose the best size and location of DGs. It was also revealed that the addition of DG to the distribution system reduces the active and reactive power loss and improves the system voltage. Particle Swarm Optimization (PSO) algorithm has been proposed by researchers [19] for optimal allocation and sizing of DGs for loss reduction. Similarly, a Multiobjective Particle Swarm Optimization (MOPSO) algorithm was used to find the optimal number, size, and location of DG units in the radial distribution systems in order to minimize the real power losses and reduce the voltage deviation in [20]. The proposed method was tested on standard IEEE 33-bus test system and it was reported that by installing DGs, the total power loss of the system was reduced and the system's voltage profile also improved. A Pareto-based Nondominated Sorting Genetic Algorithm II (NSGAII) was proposed in [21] to determine locations and sizes of specified number of DG units within the primary distribution system. In their work, three objective functions were considered as the indices of the system performance: average Load Voltage Deviation (LVD), minimization of the system real power loss, and minimization of the annualized investment costs of DG. A fuzzy decision making analysis was used to obtain the final trade-off optimal solution. The proposed methodology was tested on modified IEEE 33-bus radial system. The test results indicate that NSGA-II is a viable planning tool for practical DG placement and useful contribution of DG in improving the steady state system performance of the distribution system by the optimal allocating, setting, and sizing multitype DG.

Several algorithms have been proposed to optimize the size and the placement of DGs in a network as reviewed in the aforementioned studies. However, none considered or reported how the type of DG technologies affects the optimization problem. Different DG technologies have different reactive power characteristics which can have different effect on the power loss and voltage profile of a power system [22]. It is therefore intuitive to think that selection of appropriate DG technology alongside the optimal placement and sizing of the DG may further reduce the loss experienced in a network. This paper therefore includes DG technology as part of the optimization objective function in addition to its size and location using genetic algorithm.

\section{System under Study}

The network used to test the algorithm was the IEEE 14-bus test network as depicted in Figure 1. The data for the network were obtained from [23]. The power flow was solved using the load-flow function of Matlab based Power System Toolbox (PST) [24].

The network consists of 20 lines, 14 buses, 2 generators, 3 synchronous compensators, 10 load points, a two-winding transformer, and a three-winding transformer. The lines and the transformers were modeled using their pi-equivalent circuits while the generators and synchronous compensators were modeled using their steady state real and reactive powers as well as their reactive power generation limits. The loads were modeled using steady state values of the real and reactive power they consume.

\section{Modelling of DG Technologies}

In this paper, the DGs are modelled based on their electrical output. Three classes of DG technologies were used in the simulation and are classified as asynchronous, synchronous, and induction generator based DGs [22]. Examples of asynchronous generator based DG technologies are microturbines, fuel cells, and solar PV. They require power electronic interfaces to process the power they generate into a grid compatible one. Induction generator based DG technology includes some types of wind turbine generators (squirrel cage induction generator) that require reactive power to generate real power while synchronous generators have the capability to inject or consume reactive power. Examples of synchronous DGs include reciprocating engines, combustion turbines, and small hydroturbines. The DGs were connected to the network by appropriately modifying the bus data of the network. The data at the bus to which the DG is connected is modified using the real and reactive power values obtained from the models. The modified bus data was then used to obtain the real power loss and bus voltages of the network.

3.1. Asynchronous Generators Model. Asynchronous generators were modelled as negative loads operating at a constant power factor. Given the real power generated by the asynchronous generator based $\mathrm{DG}$ to be $P_{\mathrm{ASG}}$, then the reactive power $\left(Q_{\mathrm{ASG}}\right)$ generated by the $\mathrm{DG}$ can be written as

$$
Q_{\mathrm{ASG}}=\sqrt{P_{\mathrm{ASG}}^{2}\left(\frac{1}{\cos ^{2} \phi}-1\right)},
$$




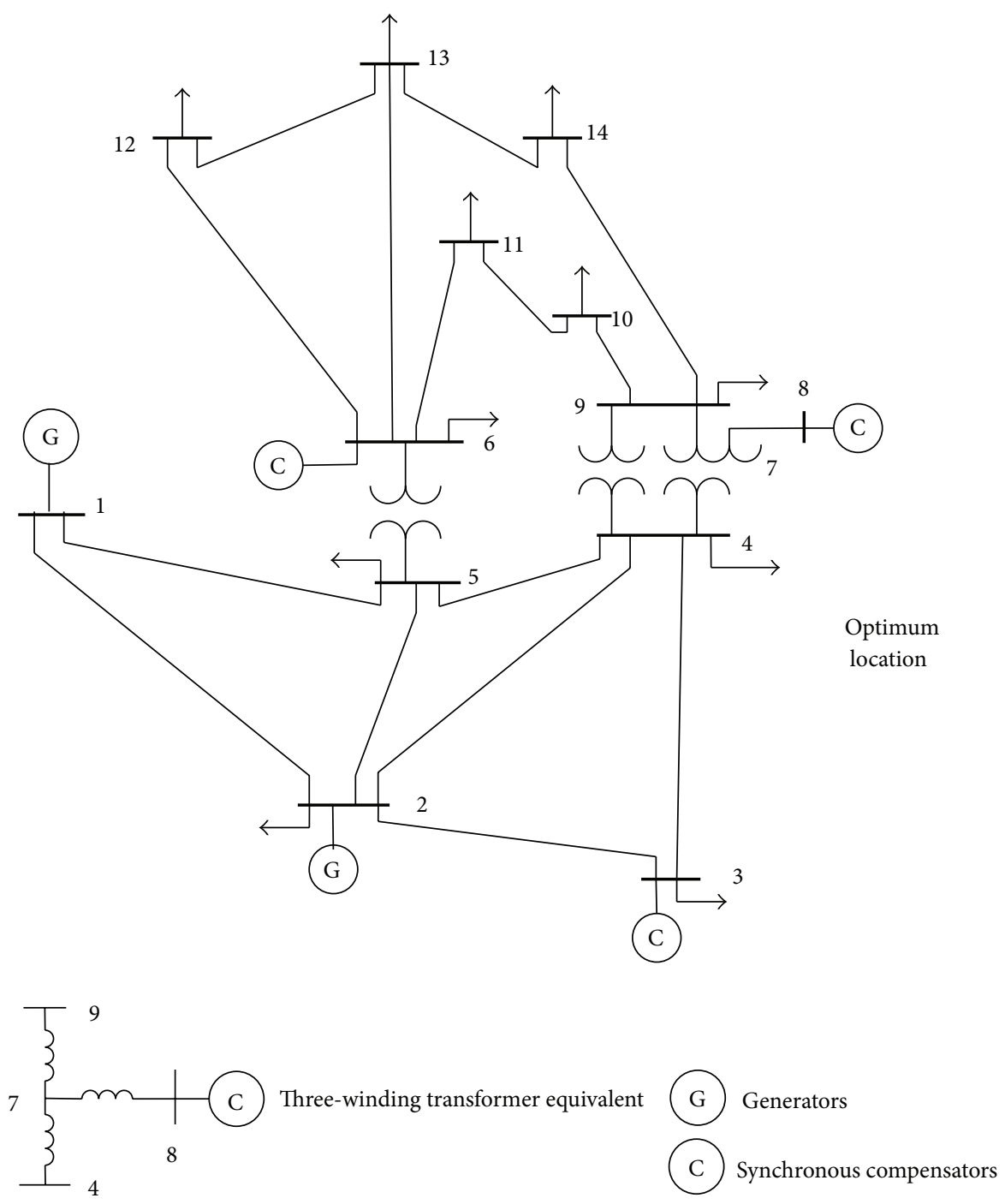

FIGURE 1: IEEE 14-bus test network.

where $\cos \phi$ is the power factor at which the DG is operating. The modelling of asynchronous generators as negative loads at load buses (PQ Buses) implies that the real and reactive power generated by the DG are fixed; however, the voltage at the bus can vary within specified limits.

3.2. Induction Generators Modelling. Induction generator based DGs are also modelled as negative loads at PQ buses; however, the induction generators require reactive power support and this is usually provided by the grid and/or capacitor banks connected at the generator's bus [25]. The reactive power $\left(Q_{\mathrm{IG}}\right)$ required for magnetization of induction generator based DG given the generated real power $P_{\mathrm{IG}}$ can be approximated as

$$
\mathrm{Q}_{\mathrm{IG}} \approx V^{2} \frac{X_{c}-X_{m}}{X_{c} X_{m}}+\frac{X}{V^{2}} P_{\mathrm{IG}}{ }^{2},
$$

where $V$ is bus voltage, $X_{m}$ is the magnetizing reactance, $X_{c}$ is the reactance of the capacitor bank, $X$ is the sum of the rotor and stator reactance, and $R$ is the sum of the stator and rotor leakage reactance.

3.3. Synchronous Generator's Model. Synchronous generator based model has the ability to maintain their terminal voltage by varying the reactive power they generate. Given that $P_{\mathrm{SG}}$ is the real power of the DG and the minimum power factor at which the DG is to operate is $\cos \phi_{\min }$, then the reactive power, $Q_{S G}$, with an upper bound $Q_{\max }$ can be determined as

$$
Q_{\max }=P_{\mathrm{SG}} \tan \phi_{\text {min }}
$$

and the lower bound $Q_{\min }$ is given by

$$
Q_{\min }=-P_{\mathrm{SG}} \tan \phi_{\min } .
$$

In view of the above, it is indicated that the generator will operate between the upper and lower limit of the reactive power; that is,

$$
-Q_{\min } \leq Q_{\mathrm{SG}} \leq Q_{\max }
$$




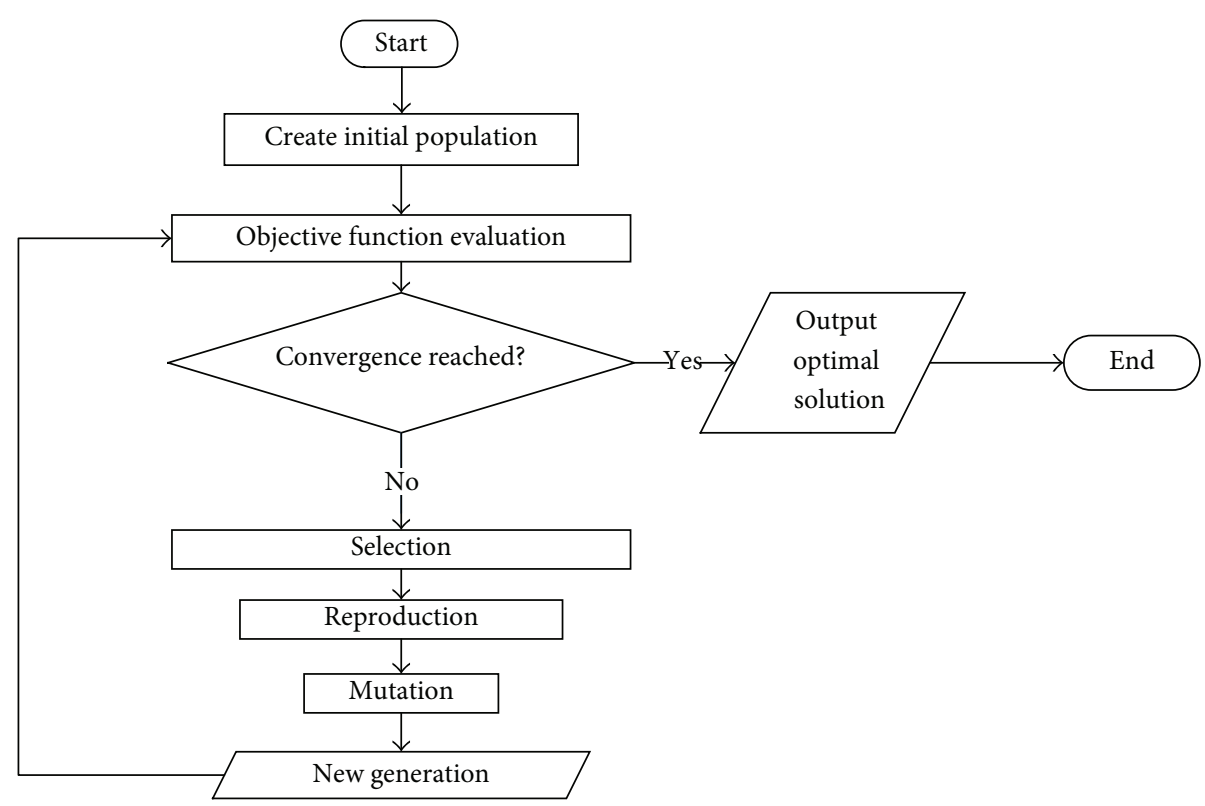

Figure 2: Genetic algorithm flow chart.

All the synchronous generators were modelled as PV buses. As a consequence, the terminal voltage is held constant and the reactive power is allowed to vary within the specified limits of the DG to maintain terminal voltage.

3.4. Determination of DG Size. The optimum sizes of DGs are determined based on the penetration level (PL) of the DG into the network. In this paper, PL is defined as the percentage of the real power demand of the load (MW) that the DGs can supply without jeopardizing the network operation [26]. This is mathematically determined using

$$
\mathrm{PL}=\frac{P_{\mathrm{DG}}}{P_{\text {load }}} \times 100 \% \text {, }
$$

where $\mathrm{PL}$ is the penetration level, $P_{\mathrm{DG}}$ is real power generated by DGs, that is, $\left(P_{\mathrm{ASG}}, P_{\mathrm{IG}}, P_{\mathrm{SG}}\right.$ depending on the technology), and $P_{\text {load }}$ is the real power demand by the load in the network.

\subsection{Development of Objective Function for Minimization of} Power Loss. The objective function is to minimize the total loss on the network. Given a transmission line between two buses $i$ and $j$, the power flow through the line from bus $i$ to bus $j$ is $S_{i j}$ and from bus $j$ to bus $i$ is $S_{j i}$. The power loss in the line can be determined as

$$
\begin{gathered}
S_{L i j}=S_{i j}+S_{j i}, \\
S_{i j}=V_{i} I^{*}{ }_{i j}, \\
S_{j i}=V I^{*}{ }_{j i},
\end{gathered}
$$

where $I$ (the current) and $V$ (the voltage at the corresponding bus) are obtained from the power flow solution of the network and $S$ is complex power flow with its real part corresponding to the real power loss on the line and the imaginary part corresponding to the reactive power loss on the line.

If there are $N$ transmission lines in the network, the total loss in the network can be calculated as

$$
M=\sum_{k=1}^{N} \operatorname{real}\left(S_{L_{k}}\right),
$$

where $k$ represents the $k$ th transmission line. The objective function therefore is to minimize $M$ subject to power flow constraints.

\section{Application of Genetic Algorithm to the Optimization Problem}

Different methods have been proposed to optimally site and locate DGs while considering different limitations, scenarios, and objectives. Three broad categories of methods are usually adopted and have been identified to be analytical methods [27], numerical approach [28], and heuristic techniques [29]. GA is one of such heuristic algorithms [28]. The GA operates by creating random solutions to the optimization problem (OP) to form a population of individuals. These individuals are then sorted based on the value they return on evaluation using the objective function. The flow chart for the GA algorithm is shown in Figure 2.

For optimal sizing and placement of the DGs for minimum power loss, the following are derived.

4.1. Initial Population. An individual solution is defined as $\left[\begin{array}{lll}x_{1} & x_{2} & x_{3}\end{array}\right]$ where $x_{1}=$ penetration level (\%) $\ni 0 \leq x_{1} \leq$ $100, x_{2}=$ location index $\ni 1 \leq x_{2} \leq L, x_{3}=$ DG Type $\ni 1 \leq$ $x_{3} \leq 3$, and $L$ is the highest location index; that is, assuming the locations considered for DG placement are numbered successively from $1, L$ is the index number of the last bus. 
$x_{3}=1$ represents asynchronous generator based DG, $x_{3}=2$ represents synchronous generator based DG, and $x_{3}=3$ represents induction generator based DG. $X:$

Let $n$ be the number of individuals in a population matrix

$$
X \triangleq\left[\begin{array}{ccc}
x_{11} & x_{12} & x_{13} \\
\vdots & \vdots & \vdots \\
x_{n 1} & x_{n 2} & x_{n 3}
\end{array}\right]
$$

The $i$ th individual is $x_{i}$ :

$$
x_{i} \triangleq\left[\begin{array}{lll}
x_{i 1} & x_{i 2} & x_{i 3}
\end{array}\right]
$$

4.2. Selection. Let $x_{i}$ and $x_{j}$ be two selected individuals from $X$ with $c_{i}$ and $c_{j}$ as the value returned by the objective function, $M$, when $x_{i}$ and $x_{j}$ are its arguments. If $P$ is the set of all parents that have a chance at reproduction, then

$$
x_{i} \in P, \quad \text { if } c_{i} \leq c_{j}
$$

4.3. Reproduction. Given a set $P$ of possible parents, let $p_{i}$ and $p_{j}$ be two selected parents, $p_{i}, p_{j} \in P$ and

$$
\begin{aligned}
& p_{i} \triangleq\left[\begin{array}{lll}
p_{i 1} & p_{i 2} & p_{i 3}
\end{array}\right], \\
& p_{j} \triangleq\left[\begin{array}{lll}
p_{j 1} & p_{j 2} & p_{j 3}
\end{array}\right] .
\end{aligned}
$$

Let $k$ be a randomly selected crossover point such that $1 \leq$ $k \leq 3$ and let $o_{1}$ and $o_{2}$ be the two crossover children from $p_{i}$ and $p_{j}$ :

$$
\begin{aligned}
& o_{1} \triangleq\left[\begin{array}{llllll}
p_{i 1} & \cdots & p_{i k} & p_{j k+1} & \cdots & p_{j 3}
\end{array}\right] \\
& o_{2} \triangleq\left[\begin{array}{lllllll}
p_{j 1} & \cdots & p_{j k} & \cdots & p_{i k+1} & \cdots & p_{i 3}
\end{array}\right] .
\end{aligned}
$$

4.4. Mutation. Given a population $X$, a mutant child $m$ is obtained by selecting a random individual $x_{i}$ from $X$. One of the genes (variables) of $x_{i}$ is randomly selected and changed. Let $j$ denote the index of the randomly selected gene.

$$
\begin{aligned}
& \text { If } j=1 \text {, then } 0 \leq x_{i j} \leq 100 . \\
& \text { If } j=2 \text {, then } 1 \leq x_{i j} \leq 3 . \\
& \text { If } j=3 \text {, then } 1 \leq x_{i j} \leq 3 .
\end{aligned}
$$

\section{Simulation Results and Discussion}

To achieve the objective function, Newton Raphson algorithm was utilized with the aid of Power System Toolbox (PST) load-flow function to obtain the power flow solution and the losses in the network. Matlab code was written to add the DG types to the network by suitably modifying the network bus data. Asynchronous generator based DGs were simulated to operate at a power factor of 1.0 while the synchronous generator based DGs were allowed to operate with a reactive power range between $-0.75 P_{\mathrm{SG}}$ and $0.75 P_{\mathrm{SG}}$. The parameters of the induction generator utilized in this paper are depicted in Table 3.
After running the algorithm, the returned optimal solution $\left(x_{\text {opt }}\right)$ was given as $\left[\begin{array}{lll}71 & 1 & 4\end{array}\right]$ to minimize the real power losses in the network. The physical meaning of this is that a $71 \%$ penetration level of synchronous generator based DG technology operating at bus 4 will result in the best reduction in losses as depicted in Figure 3.

To verify the optimality of the solution, three tests were performed. Test 1 was done to confirm that bus 4 is the optimal location. Test 2 was to confirm that synchronous generator based DGs produce the least loss in the network and Test 3 was done to confirm that the optimal penetration level is $71 \%$.

5.1. Convergence Characteristics. Figure 4 depicts the best and mean cost obtained with successive generations using a population size of 45 and a crossover fraction of 0.8 . From the figure it could be observed that the mean cost decreases rapidly in the first few generations. However, as the number of generations increases the mean cost starts to oscillate. This is largely due to the random variables introduced by mutation. In this particular run of the algorithm, the best cost converges after 14 generations.

5.2. Test 1: Confirmation of Optimum Location. To achieve this, synchronous generator based DG model was used and the penetration level was varied between 50 and $90 \%$ at all the PQ buses. The results are presented in Figure 5. The figure reveals that bus 4 has the least power loss at all the penetration levels.

5.3. Test 2: Confirmation of the Most Appropriate DG Technology. To determine which type of DG results in the minimum loss, the three DG models were placed at bus 4 and the amount of power they produced was increased by varying the penetration from 50 to $90 \%$. The power losses incurred as a result of the connection of each generator type were calculated for different penetration level and the result is depicted in Figure 6.

It is observed from the figure that the losses when synchronous and induction generators used are practically the same up to penetration level of $71 \%$. However, as penetration levels increase, the power loss when synchronous generator technologies are used are lower, compared to the induction or asynchronous generator based DGs which are used. Asynchronous generators do not offer as much loss reduction as synchronous generator or induction generator based DGs. On the overall, synchronous generator technology offer the best reduction in network losses at bus 4 .

5.4. Test 3: Confirmation of DG Penetration Level (Size). This scenario involved using a synchronous generator based DG at bus 4 to find the penetration level at which loss is minimum. The result is shown in Figure 7. It can be seen that the minimum loss is obtained at the penetration levels of about $71 \%$ confirming the size of the DG in terms of penetration level.

The three tests confirm that the location, size, and type of $\mathrm{DG}$ required to obtain the least loss is bus 4 with 


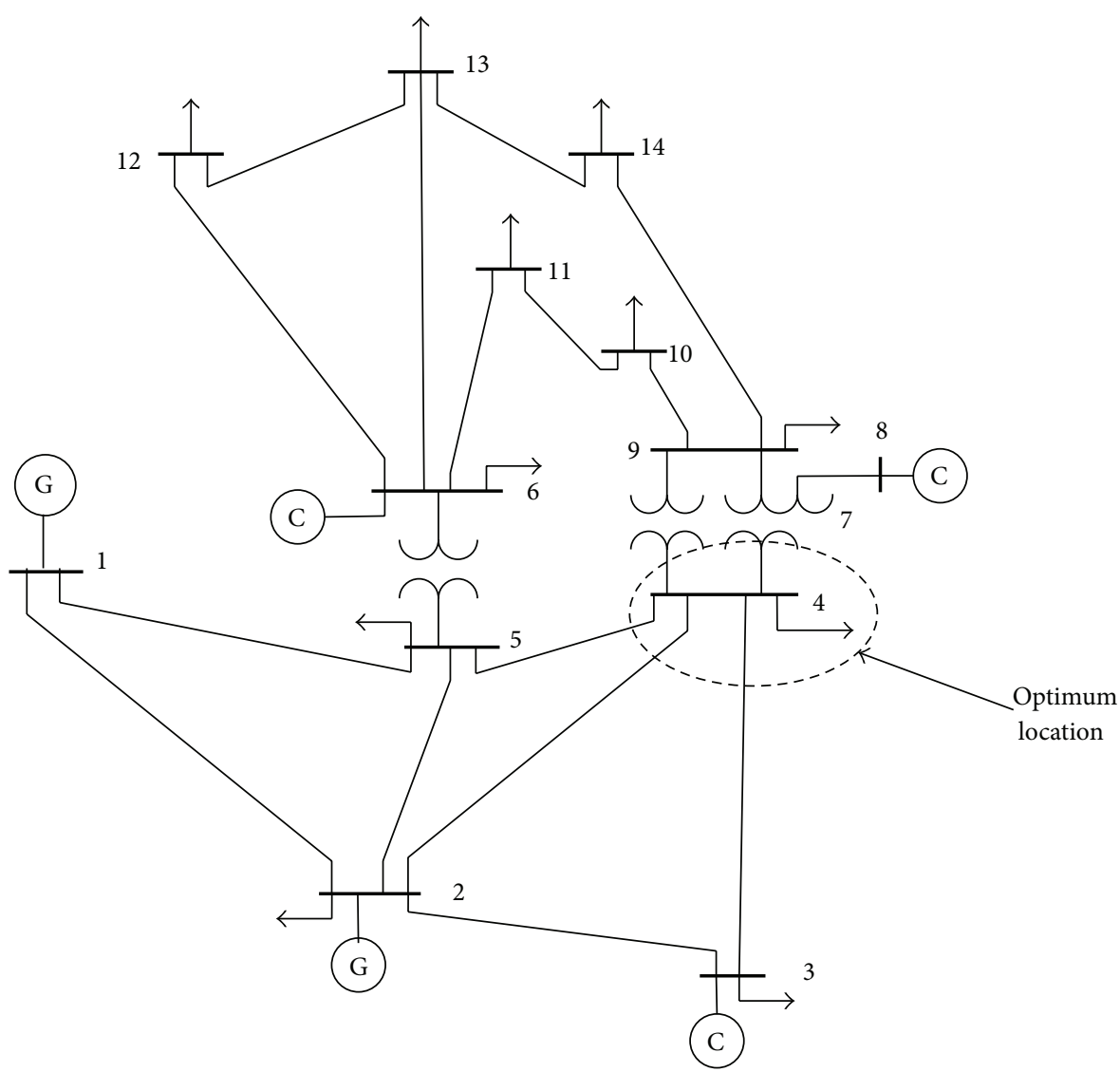

FIGURE 3: Optimal location of the DG in the IEEE 14-bus test network.

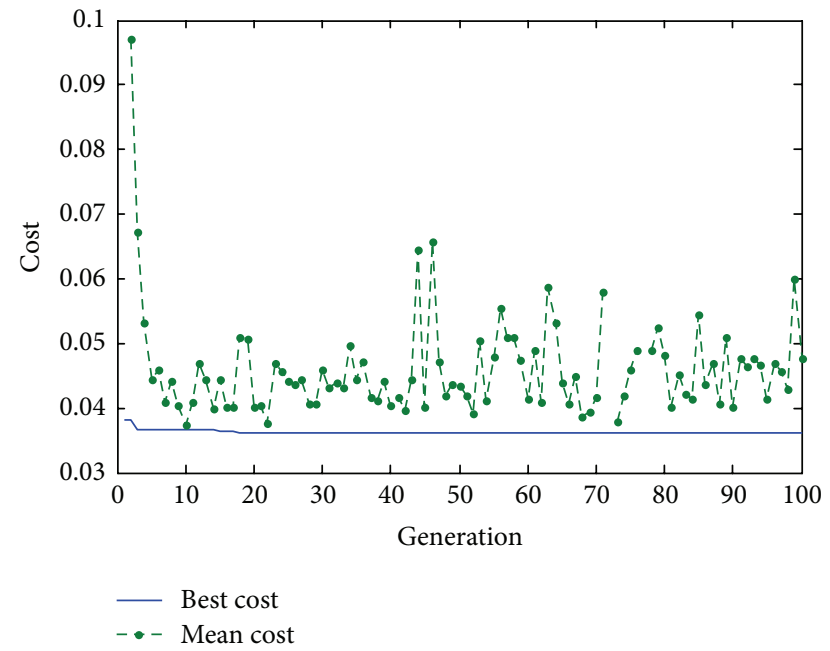

FIGURE 4: Convergence characteristics.

a synchronous generator based DG operating at a penetration level of $71 \%$.

5.5. Multiple DG Location for Power Loss Reduction. To check for further reduction in losses if multiple DGs are installed in the network, the bus data of the network was modified

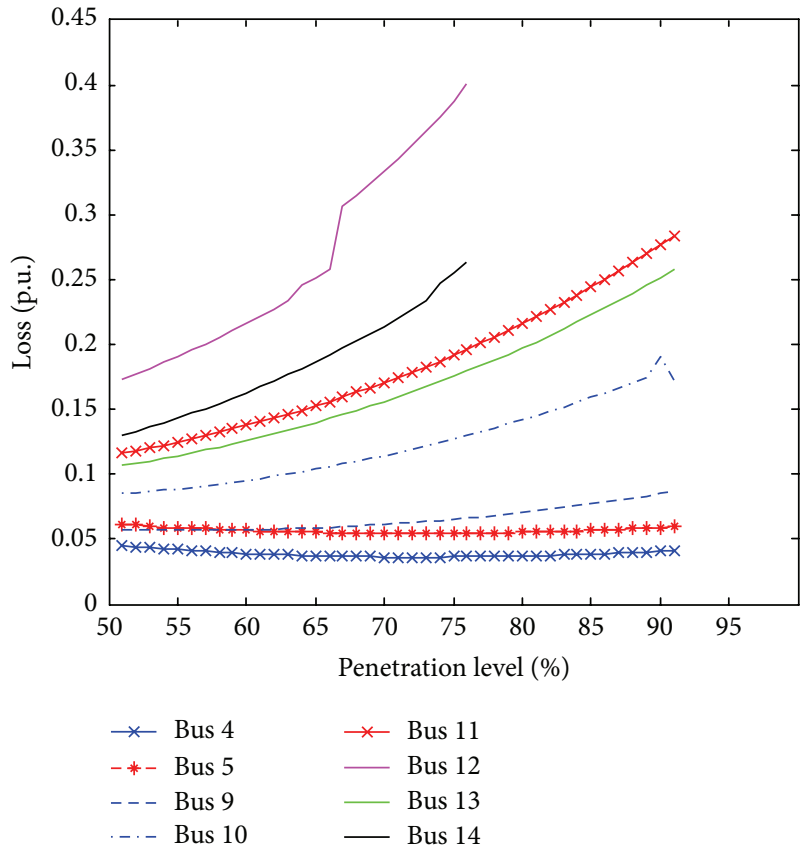

FIgURE 5: Loss at all the PQ buses for DG placement.

to include the previous solution and then inputted into the GA algorithm to determine another location, penetration 


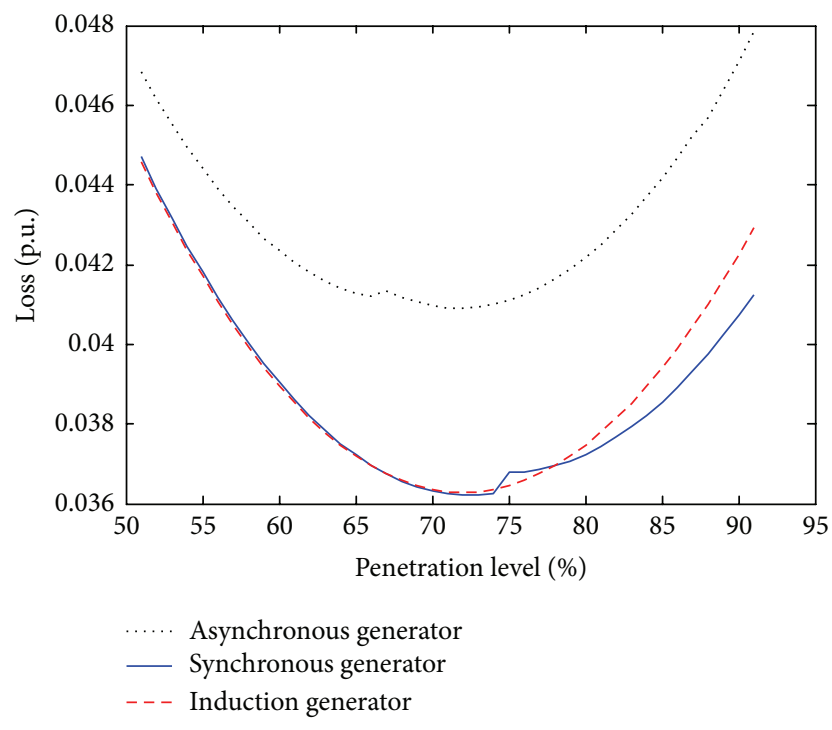

Figure 6: Loss obtainable from using different technologies.

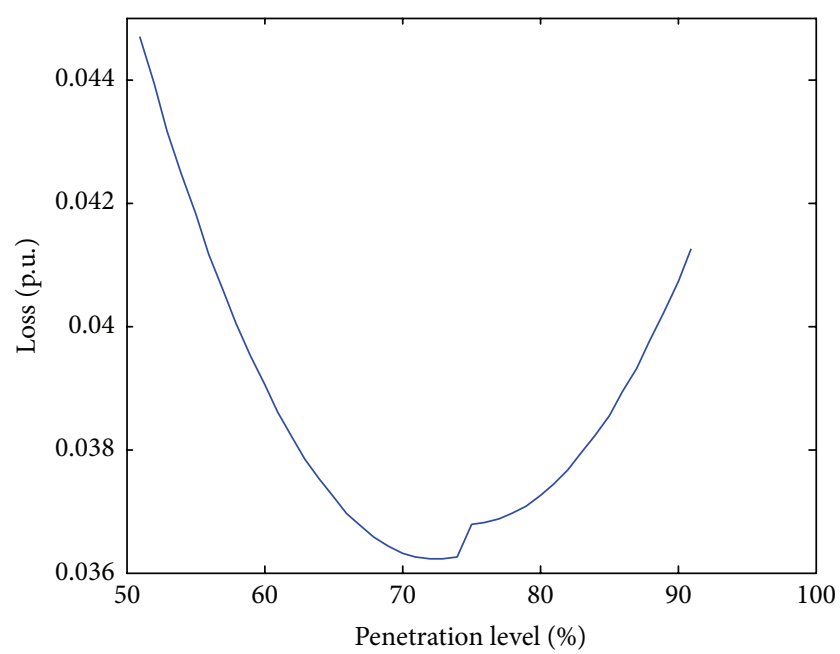

FIGURE 7: Loss as penetration level varies at bus 4 with a synchronous generator based DG.

level, and DG type that will result in further loss reduction. The result is presented in Figure 8. From the figure, it is observed that placing the DG at additional buses (multiple DGs) yielded a further reduction in the loss. Bus number $4+$ 14 as labelled in Figure 8 indicates that DG is located at buses 4 and 14 while $4+14+13$ indicates that the DG is located at buses 4,14 , and 13 .

The locations of the multiple DGs on the network for loss reduction are depicted in Figure 9. These four locations (in group) represent the possible optimal points for the placement of DGs. Placement at additional bus(es) did not improve the line losses beyond those displayed in Figure 9.

The penetration level and the technology type to obtain further reduce losses in the network at each of the placement is depicted in Table 1.

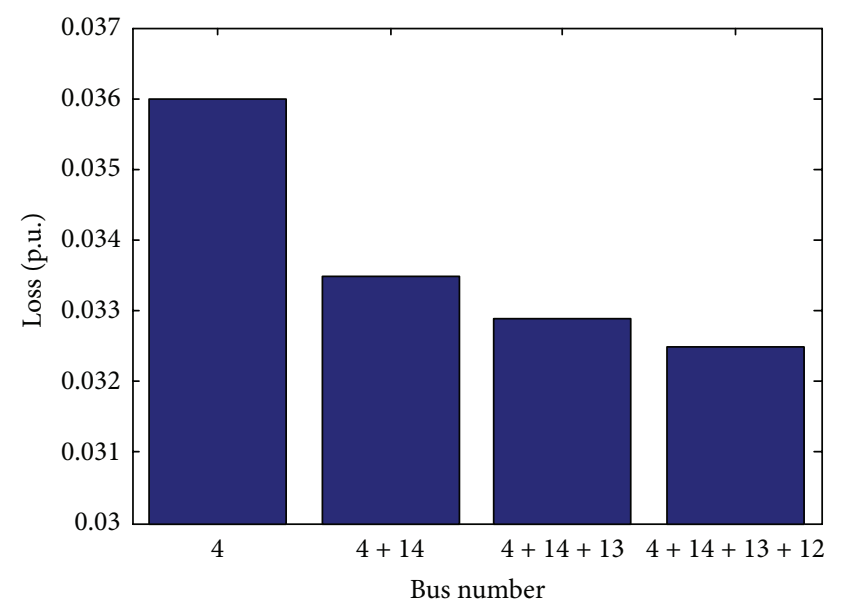

FIGURE 8: Minimum loss obtainable with multiple DG placements.

TABLE 1: Placement order of the DGs to achieve reduction in losses.

\begin{tabular}{lcc}
\hline Placement & Penetration level (\%) & Technology type \\
\hline Bus 4 & 71 & Synchronous \\
Bus 14 & 7 & Synchronous \\
Bus 13 & 4 & Asynchronous \\
Bus 12 & 1 & Synchronous \\
\hline
\end{tabular}

TABLE 2: Percentage loss reduction in the network with multiple placements.

\begin{tabular}{lc}
\hline Placement of DG at the buses & Loss reduction (\%) \\
\hline 4 & 73.39 \\
$4+14$ & 75.74 \\
$4+14+13$ & 75.91 \\
$4+14+13+12$ & 75.98 \\
\hline
\end{tabular}

TABLE 3: Induction generator parameters.

\begin{tabular}{lc}
\hline Parameter & Value \\
\hline Stator reactance & 0.01 p.u. \\
Rotor reactance & 0.01 p.u. \\
Magnetizing reactance & 3.0 p.u. \\
\hline
\end{tabular}

Table 2 presents the percentage loss reduction when multiple DGs are placed in the network. When DG is placed only on bus 4 , the percentage loss reduction is $73.39 \%$. When the number of placements of DG is increased (i.e., buses 4 and 14), an improved loss reduction of $75.74 \%$ is achieved. However, the reduction gained with the increase in the number of DG locations becomes smaller indicating that at certain number of placements, a further increase in the number of placements will not improve the line loss.

\section{Conclusion}

Most algorithms proposed in the literature to solve optimal DG placement problems consider only location and size as 


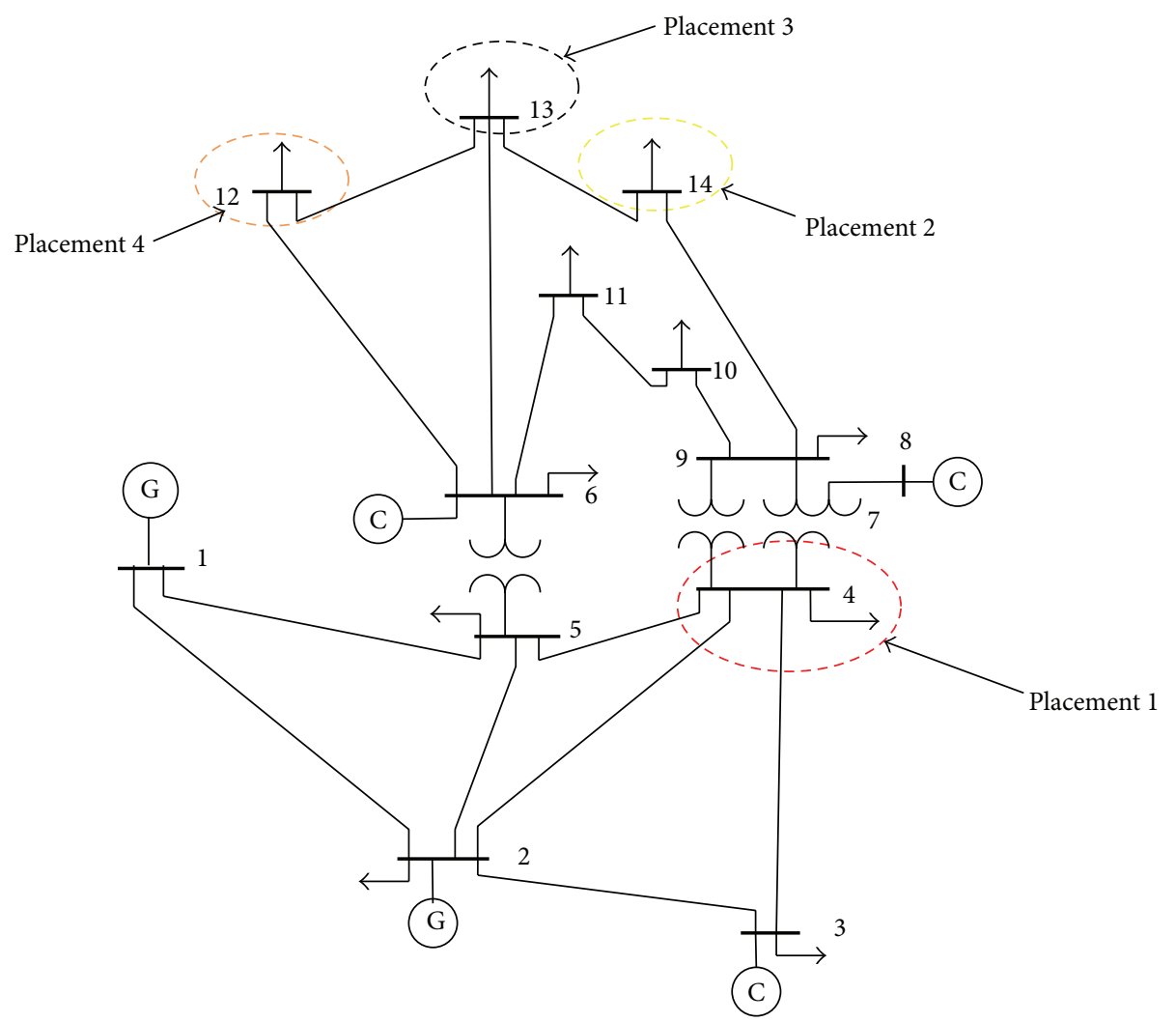

FIGURE 9: Multiple DG placements in IEEE 14-bus network.

the variables of optimization in minimizing the power loss in a network. However, DG technology also plays an important role in minimizing the loss on the network. This paper has developed a genetic algorithm having DG technology as a third variable in addition to size and location to minimize loss on the power system. The developed algorithm successfully optimized the location and size (penetration level) of the DG and determines the appropriate DG technology. It was further shown that the algorithm was able to optimally locate and size more DGs to further reduce losses on the network.

\section{Appendix}

See Table 3.

\section{Conflict of Interests}

The authors declare that there is no conflict of interests regarding the publication of this paper.

\section{References}

[1] S. D. M. Shareef and V. T. Kumar, "A review on models and methods for optimal placement of distributed generation in power distribution system," International Journal of Education and Applied Research, vol. 4, no. 1, pp. 161-169, 2014.

[2] R. Ishak, A. Mohamed, A. N. Abdalla, and M. Z. C. Wanik, "Optimal DG placement and sizing for voltage stability improvement using backtracking search algorithm," in Proceedings of the International Conference on Artificial Intelligence, Energy and Manufacturing Engineering (ICAEME '14), pp. 2934, Kuala Lumpur, Malaysia, June 2014.

[3] P. Chiradeja, "Benefit of distributed generation: a line loss reduction analysis," in Proceedings of the IEEE/PES Transmission and Distribution Conference and Exhibition: Asia and Pacific, pp. 1-5, IEEE, Dalian, China, August 2005.

[4] J. A. Peças Lopes, "Integration of dispersed generation on distribution networks-impact studies," in Proceedings of the IEEE Power Engineering Society Winter Meeting, pp. 323-328, IEEE, January 2002.

[5] P. Chiradeja and R. Ramakumar, "An approach to quantify the technical benefits of distributed generation," IEEE Transactions on Energy Conversion, vol. 19, no. 4, pp. 764-773, 2004.

[6] F. H. Guan, D. M. Zhao, X. Zhang, B. T. Shan, and Z. Liu, "Research on distributed generation technologies and its impacts on power system," in Proceedings of the 1st International Conference on Sustainable Power Generation and Supply (SUPERGEN '09), pp. 1-6, Nanjing, China, April 2009.

[7] G. P. Harrison, A. Piccolo, P. Siano, and A. R. Wallace, "Hybrid GA and OPF evaluation of network capacity for distributed generation connections," Electric Power Systems Research, vol. 78, no. 3, pp. 392-398, 2008.

[8] G. Pepermans, J. Driesen, D. Haeseldonckx, R. Belmans, and W. D'haeseleer, "Distributed generation: definition, benefits and issues," Energy Policy, vol. 33, no. 6, pp. 787-798, 2005.

[9] L. F. Ochoa, A. Padilha-Feltrin, and G. P. Harrison, "Evaluating distributed generation impacts with a multiobjective index," 
IEEE Transactions on Power Delivery, vol. 21, no. 3, pp. 1452$1458,2006$.

[10] Q. Sun, Z. Li, and H. Zhang, "Impact of distributed generation on voltage profile in distribution system," in Proceedings of the International Joint Conference on Computational Sciences and Optimization (CSO '09), pp. 249-252, Sanya, China, April 2009.

[11] P. K. Kumar, "Selection of optimal location and size of multiple distributed generations by using kalman filter algorithm," International Journal of Engineering Research and Applications, vol. 4, no. 3, pp. 1708-1729, 2013.

[12] P. S. Rani and A. L. Davi, "Optimal sizing of DG units using exact loss formula at optimal power factor," International Journal of Engineering Science and Technology, vol. 4, no. 9, pp. 40434050, 2012.

[13] R. M. Kamel and B. Kermanshahi, "Optimal size and location of distributed generations for minimizing power losses in a primary distribution network," Scientia Iranica, vol. 16, no. 2, pp. 137-144, 2009.

[14] M. J. Kasaei, "Optimal placement of distributed generation and capacitor in distribution networks by ant colony algorithm," International Journal on Technical and Physical Problems of Engineering, vol. 6, no. 20, pp. 52-56, 2014.

[15] S. A. A. Shahriari and H. Samet, "Optimal allocation of fault current limiters and distributed generations in the presence of remote controllable switches," Journal of Electrical Systems, vol. 10, no. 2, pp. 149-155, 2014.

[16] V. V. Nagireddy, D. V. A. Kumar, and K. V. Reddy, "Optimal placement and sizing of multiple distributed generation using combined differential evaluation-HPSO method," International Journal of Engineering and Advanced Technology, vol. 4, no. 1, pp. 2249-2256, 2014.

[17] R. Ishak, A. Mohamed, A. N. Abdalla, and M. Z. C. Wanik, "Optimal DG placement and sizing for voltage stability improvement using backtracking search algorithm," in Proceedings of the International Conference on Artificial Intelligence, Energy and Manufacturing Engineering, pp. 29-34, Kuala Lumpur, Malaysia, 2014.

[18] M. F. Kotb, K. M. Shebl, M. El Khazendar, and A. El Husseiny, "Genetic algorithm for optimum siting and sizing of distributed generation," in Proceedings of the 14th International Middle East Power Systems Conference (MEPCON '10), Cairo, Egypt, December 2010.

[19] K. R. Guerriche and T. Bouktir, "Optimal allocation and sizing of distributed generation with particle swarm optimization algorithm for loss reduction," Revue des Sciences et de la Technologie, vol. 6, no. 1, pp. 59-69, 2015.

[20] S. Molazei, "Maximum loss reduction through DG optimal placement and sizing by MOPSO algorithm," Recent Researches in Environment and Biomedicine, vol. 4, pp. 143-147, 2010.

[21] K. Buayai, "Optimal multi-type DGs placement in primary distribution system by NSGA-II," Research Journal of Applied Sciences, Engineering and Technology, vol. 4, no. 19, pp. 36103617, 2012.

[22] C. J. Mozina, "A tutorial on the impact of distributed generation (DG) on distribution systems," in Proceedings of the 61st Annual Conference for Protective Relay Engineers, pp. 591-609, College Station, Tex, China, April 2008.

[23] F. Milano, Power System Modelling and Scripting, Springer, Berlin, Germany, 2010.

[24] J. Chow and G. Rogers, Power System Toolbox Version 3.0, Graham-Rogers, Ontario, Canada, 2008.
[25] A. E. Feijöo and J. Cidrás, "Modeling of wind farms in the load flow analysis," IEEE Transactions on Power Systems, vol. 15, no. 1, pp. 110-115, 2000.

[26] F. M. Gonzalez-Longatt, "Impact of distributed generation over power losses on distribution system," in Proceedings of the 9th International Conference, Electrical Power Quality and Utilisation, Barcelona, Spain, 2007.

[27] C. Wang and M. H. Nehrir, "Analytical approaches for optimal placement of distributed generation sources in power systems," IEEE Transactions on Power Systems, vol. 19, no. 4, pp. 20682076, 2004.

[28] P. S. Georgilakis and N. D. Hatziargyriou, "Optimal distributed generation placement in power distribution networks: models, methods, and future research," IEEE Transactions on Power Systems, vol. 28, no. 3, pp. 3420-3428, 2013.

[29] R. K. Singh and S. K. Goswami, "Optimum siting and sizing of distributed generations in radial and networked systems," Electric Power Components and Systems, vol. 37, no. 2, pp. 127$145,2009$. 


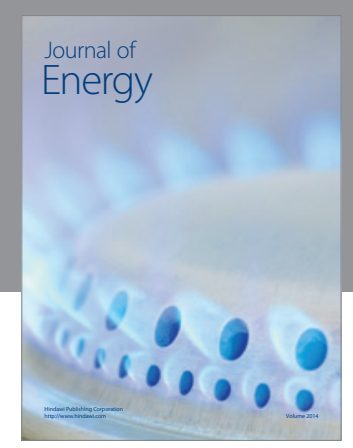

Journal of

Industrial Engineering
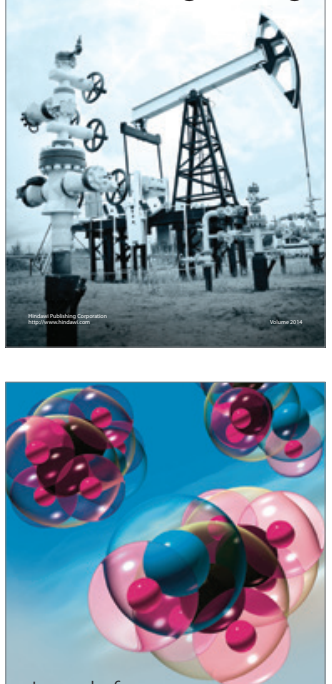

Fuels
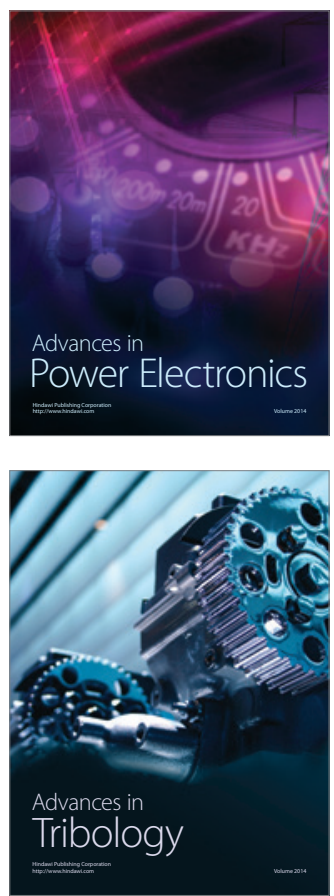

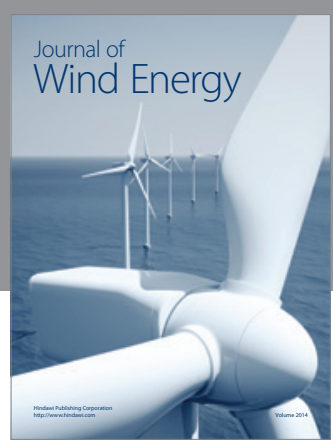

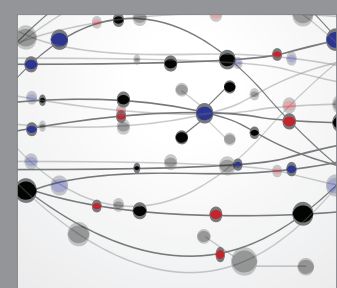

The Scientific World Journal

Submit your manuscripts at http://www.hindawi.com

Journal of

Structures
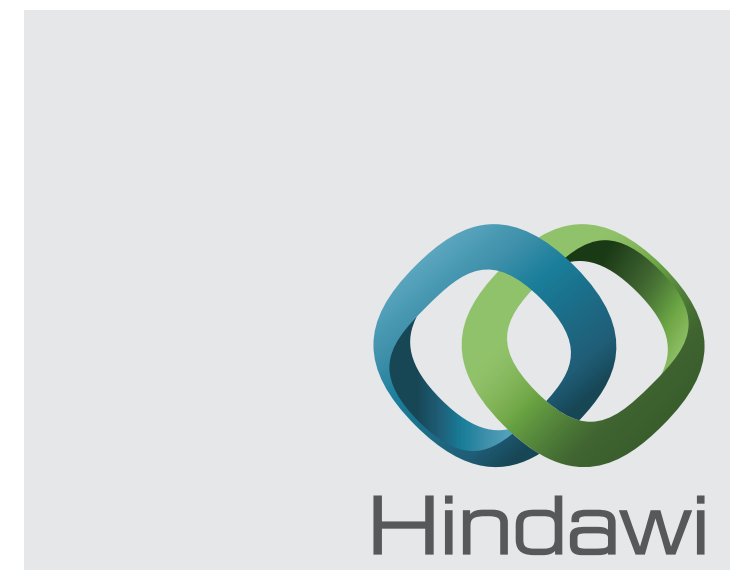

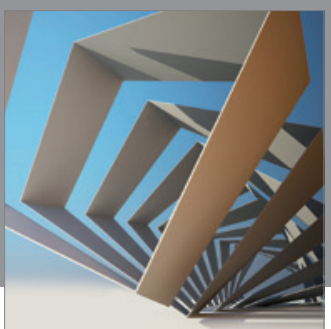

Rotating

Machinery
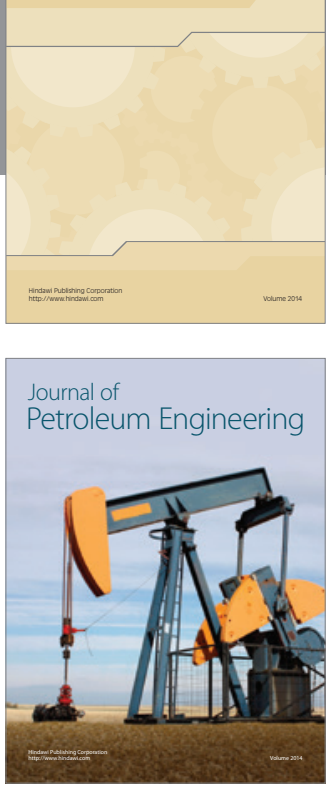

Journal of

Solar Energy
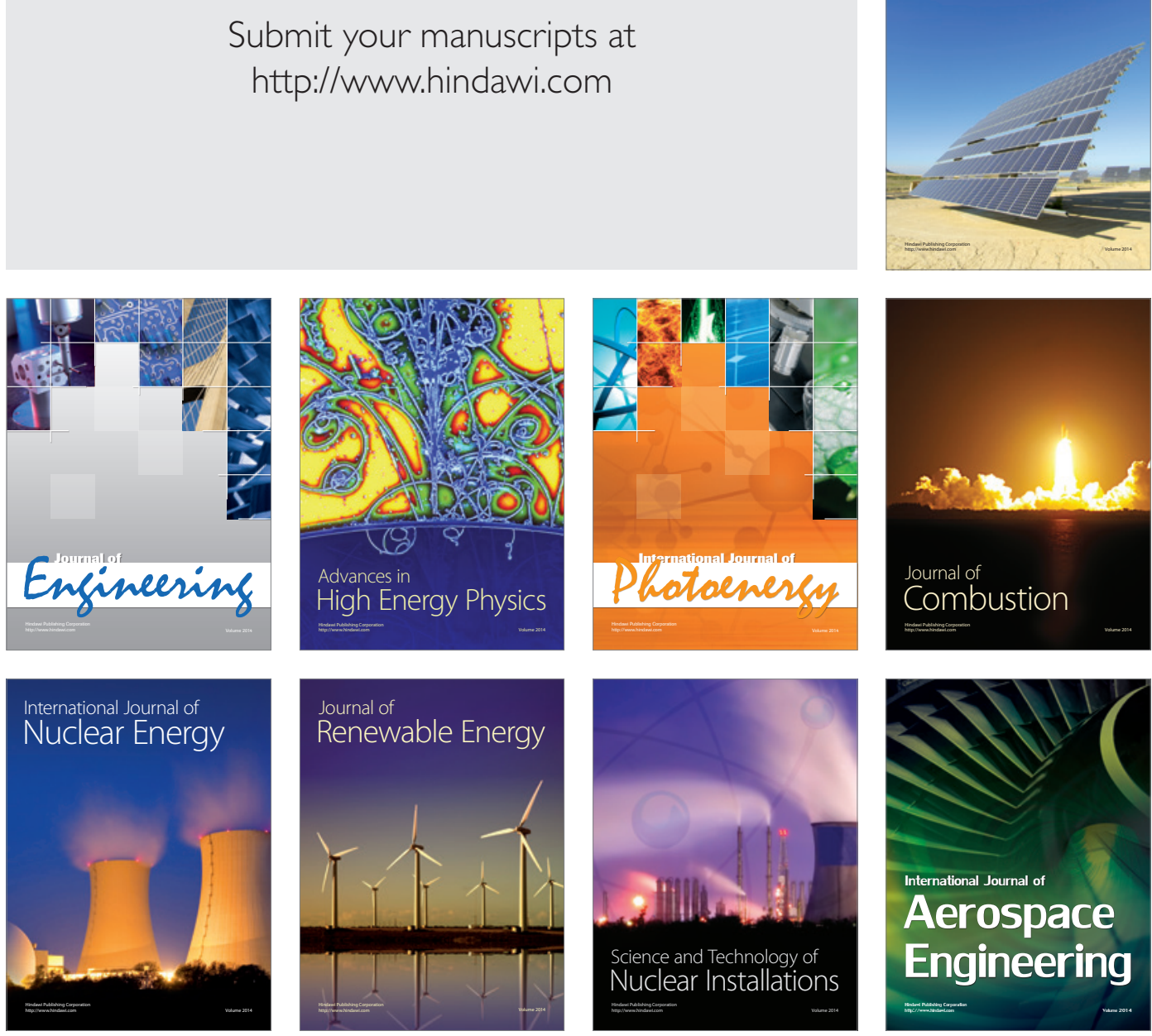